\title{
Theoretical Analysis of Pulse Dynamics in Silicon Photonic Crystal Wire Waveguides
}

\author{
Nicolae C. Panoiu, James F. McMillan, and Chee Wei Wong, Member, IEEE
}

\begin{abstract}
In this paper, we present a comprehensive theoretical description of the propagation of optical pulses in 1-D waveguides consisting of a line defect in a photonic crystal (PhC) slab waveguide made of silicon. We incorporate in our analysis linear optical effects, such as group-velocity dispersion and optical losses, as well as nonlinear effects induced by the Kerr nonlinearity of the PhC. We also include in our model the free-carrier (FC) dispersion and FC-induced optical losses, and thus study the influence of FCs generated through two-photon absorption on the pulse dynamics. Our analysis reveals that important quantities, such as the pulse group velocity, dispersion coefficients, or the waveguide nonlinear coefficient are strongly affected by the periodic nature of the guiding structure. Finally, we demonstrate that both linear and nonlinear effects are stronger in the case of slow-light modes, with the nonlinear effects being enhanced more as compared to the linear ones.
\end{abstract}

Index Terms-Coupled-mode analysis, nonlinear wave propagation, photonic bandgap materials.

\section{INTRODUCTION}

$\mathbf{R}$ ECENTLY, we have witnessed a dramatic growth in the research on passive and active optoelectronic devices based on Si [1], [2]. There are several factors that have sparked this renewed interest in silicon photonics. For example, Si-based photonic devices can be manufactured using the well-established low-cost mass-production CMOS process technology, and as such a seamless integration with current technologies can be readily achieved. Furthermore, there are several unique properties of Si that make it an ideal integration medium for functional photonic devices. First, by employing a Si core $\left(n_{\mathrm{Si}}=3.45\right)$ with air $(n=1)$ or silica $\left(n_{\mathrm{SiO}_{2}}=1.45\right)$ cladding, one can achieve a very tight field confinement and, consequently, enhanced power flux. Second, related to the just mentioned tight guiding property, high-index contrast guiding structures provide a convenient means to strongly enhance nonlinear optical processes and thus achieve a higher degree of device integration. This property is particularly important in the case of $\mathrm{Si}$ as the values of its nonlinear optical constants are large as compared to those of many other optical media. Equally important,

Manuscript received May 1, 2009; revised June 3, 2009. First published January 15, 2010; current version published February 5, 2010. This work was supported by the Engineering and Physical Sciences Research Council (EPSRC) under Grant EP/G030502/1.

N. C. Panoiu is with the Photonics Group, Department of Electronic and Electrical Engineering, University College London, London WC1E 7JE, U.K. (e-mail: npanoiu@ee.ucl.ac.uk).

J. F. McMillan and C. W. Wong are with the Optical Nanostructures Laboratory, Columbia University, New York, NY 10027 USA (e-mail: jfm2113@ columbia.edu; cww2104@columbia.edu).

Color versions of one or more of the figures in this paper are available online at http://ieeexplore.ieee.org.

Digital Object Identifier 10.1109/JSTQE.2009.2025392 for subwavelength guiding structures, both the linear dispersion coefficients [3]-[5], such as the effective index, the group velocity $(\mathrm{GV})$, or the group-velocity dispersion (GVD), as well as the nonlinear effective coefficient [6] depend chiefly on the geometry of the structure.

Although Si photonics could have a significant impact on a series of current technologies, it is envisioned that its most far-reaching applications will be the in area of ultrafast optical interconnects. Thus, similar to optical-fibers-based communication systems, which use optical pulses as fundamental carriers of information, it is envisioned that the backbone of future onchip optical networks will consist of Si subwavelength guiding structures that would generate, modulate, amplify, and transmit high bit-rate data streams, leading to a monolithic integration of electronics and photonics in the same Si chip. In this scheme, the signal processing is performed at the chip level, and therefore, Si-based active devices involving nonlinear optical effects would play a central role. To this end, important nonlinear effects in Si wire waveguides, such as light amplification [7]-[10], optical modulation [11], [12], self-phase modulation (SPM) [13], [14], cross-phase modulation (XPM) [15], [16], four-wave mixing [17], [18], or modulation instability [19], have been already demonstrated (for a review of nonlinear optical phenomena in Si wires, see [6], [20], and [21]).

One very promising approach to significantly decrease the footprint of Si-based photonic circuits is to employ nanostructured devices, e.g., waveguides based on photonic crystals $(\mathrm{PhCs})$. Subwavelength patterning increases the set of parameters that determine the properties of a photonic structure, and thus, it provides an efficient approach to optimize its functionality. In addition, it is possible to tune the geometrical parameters of the $\mathrm{PhC}$ so that it supports slow-light optical modes with GV, $v_{g}$, as small as $10^{-4} c$. As a result, nonlinear effects are dramatically enhanced [22]-[24] and, due to the small nonlinear length, the footprint of active devices is significantly reduced. For example, it has been shown recently that in the slow-light regime, nonlinear effects, such as Raman interaction [25], [26], third-harmonic generation [27], [28], or superprism effects [29], can be dramatically enhanced.

Achieving an in-depth understanding of the effects that determine the dynamics of optical pulses (data carriers) upon propagation in deep subwavelength, Si-based guiding devices is a major milestone on the path to a successful on-chip implementation of the functionality of ultrafast optical interconnects based on $\mathrm{Si}$. In this paper, we address this important problem and develop a comprehensive theoretical model that accurately describes the propagation of optical pulses in $\mathrm{Si} \mathrm{PhC} \mathrm{waveguides} \mathrm{(} \mathrm{Si}-\mathrm{PhCW}$ ). Our analysis accounts both for the dynamics of the optical field 


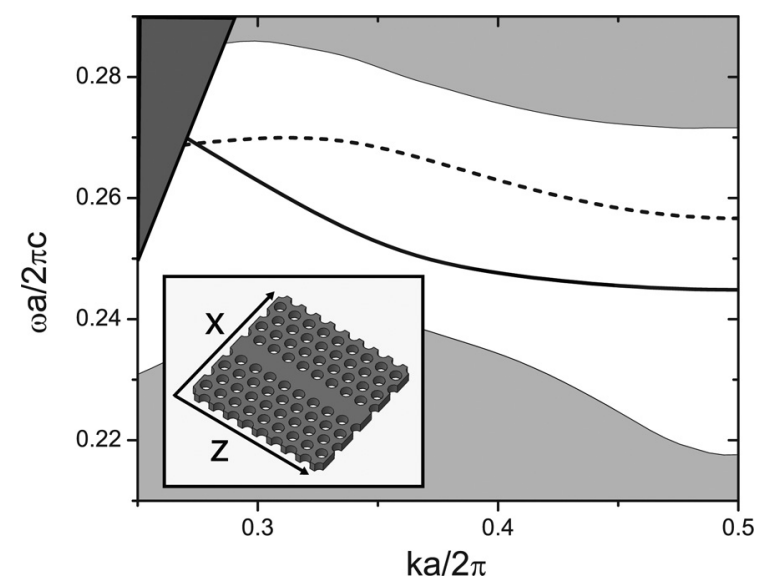

Fig. 1. Projected band structure of a Si PhC slab waveguide with $h=0.6 a$ and $r=0.22 a$. Solid and dashed curves correspond to the fundamental (even) and second (odd) mode, respectively. Light gray and dark gray regions correspond to slab guiding modes and leaky modes, respectively.

as well as its interaction with the generated free-carriers (FCs), and thus provides a complete description of a series of linear and nonlinear effects pertaining to the optical pulse propagation in such subwavelength guiding structures.

The paper is organized as follows. In the next section, we describe the geometry and optical properties of Si-PhCWs. Then, in Section III, we derive the coupled-mode equations describing the pulse propagation in $\mathrm{Si}-\mathrm{PhCW}$ as well as the pulse interaction with the FCs. In Section IV, we apply our general theory on two particular cases, namely, pulse propagation in a singlemode waveguide and interaction of two pulses that propagate in different waveguide modes. In the last section, we summarize our main results.

\section{Device Description: Structure and Optical-Mode CHARACTERISTICS}

The waveguide considered in this study consists of a 1-D line defect in a PhC slab waveguide (see Fig. 1). The PhC consists of a Si slab with thickness $h$, perforated by a hexagonal lattice of holes, the lattice constant and hole radius being $a$ and $r$, respectively. The line defect is obtained by filling one of the rows of holes that are oriented along the KГ crystal symmetry axis. The $\mathrm{PhC}$ slab is located in the $x-z$ plane, with the coordinate axes being chosen such that the line defect is along the $z$-axis. Then, the spatial distribution of the index of refraction is described by the function $n(\mathbf{r})$, where $n(\mathbf{r})=1$ for $\mathbf{r}$ corresponding to the holes or outside the slab, and $n(\mathbf{r})=$ $n_{\mathrm{Si}} \equiv n$ for $\mathbf{r}$ corresponding to the $\mathrm{Si}$ regions (note that we use the same symbol for the spatial distribution of the index of refraction and the index of refraction of $\mathrm{Si}$, but the meaning of the symbol should be clear from the context).

The photonic band structure corresponding to the $\mathrm{PhC}$ waveguide has been determined by using a numerical method based on the plane-wave expansion algorithm [30]. We have considered a PhC waveguide with $h=0.6 a$ and $r=0.22 a$, and a supercell is used in our numerical simulations with a size of $6 \sqrt{3} a \times 4 a \times a$ along the $x-, y$-, and $z$-axis, respectively. The
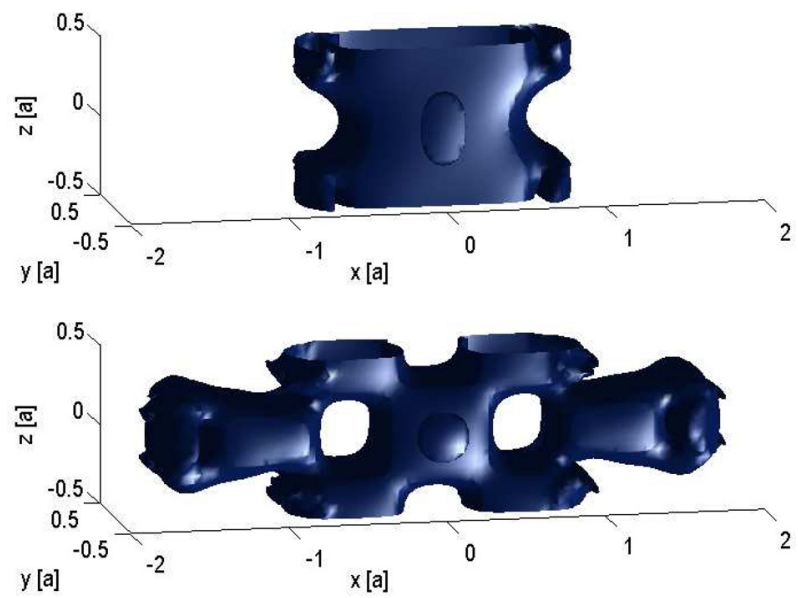

Fig. 2. Isosurface plots of the field intensity $|\mathbf{E}(\mathbf{r})|^{2}$ corresponding to a mode with $\tilde{\omega}=0.267$ (top panel) and a slow-light mode with $\tilde{\omega}=0.245$ (bottom panel).

computational step size along the $x, y$, and $z$ directions was $a \sqrt{3} / 40, a / 20$, and $a / 20$, respectively. The results of our numerical simulations, presented in Fig. 1, show that the $\mathrm{PhC}$ waveguide has two guiding modes located in the frequency bandgap of the $\mathrm{PhC}$ slab waveguide, both of these modes being TE-like modes. The fundamental mode is even upon reflection transformations with respect to the $y-z$ plane, whereas the other mode is odd with respect to such symmetry transformations. Since the spatial distribution of the index of refraction $n(\mathbf{r})$ of the $\mathrm{PhC}$ waveguide is periodic along the $z$-axis, the Bloch theorem implies that the mode propagation constant $\beta$, which is oriented along the $z$-axis, is restricted to the first Brillouin zone, $\beta \in[-\pi / a, \pi / a]$. Also note that the dispersion curves shown in Fig. 1 are represented in dimensionless units, namely, $\tilde{\omega}=\omega a / 2 \pi c$ for frequency and $\tilde{k}=\beta a / 2 \pi$ for the wave vector. Importantly, the dispersion curves in Fig. 1 do not include the material dispersion, i.e., $n(\mathbf{r})$ does not depend on $\omega$. The material dispersion can be readily incorporated in our analysis by using a frequency-dependent index of refraction of $\mathrm{Si}$

$$
n^{2}(\lambda)=\epsilon+\frac{A}{\lambda^{2}}+\frac{B \lambda_{1}^{2}}{\lambda^{2}-\lambda_{1}^{2}} .
$$

For silicon, the material constants in (1) are $\lambda_{1}=1.1071 \mu \mathrm{m}$, $\epsilon=11.6858, A=0.939816 \mu \mathrm{m}^{2}$, and $B=8.10461 \times 10^{-3}$. However, note that for subwavelength guiding structures, the waveguide dispersion is much larger than the material dispersion, and therefore, the latter one can be neglected.

The fact that the mode dispersion is chiefly determined by the waveguide dispersion can be easily understood if one considers the field distribution of the waveguide modes. Thus, as Fig. 2 shows, in the case of $\mathrm{PhC}$ waveguides, the field profile is strongly dependent on the mode frequency $\omega$, and therefore, one expects that the propagation constant changes markedly with $\omega$. In addition, it can be seen that as the mode approaches the slowlight regime, its spatial extent increases significantly. The dependence $\beta=\beta(\omega)$ allows us to determine a set of waveguide dispersion coefficients that characterize the optical pulse dispersion in the waveguide. Thus, $\beta_{1} \equiv \partial \beta / \partial \omega=1 / v_{g}$ defines 


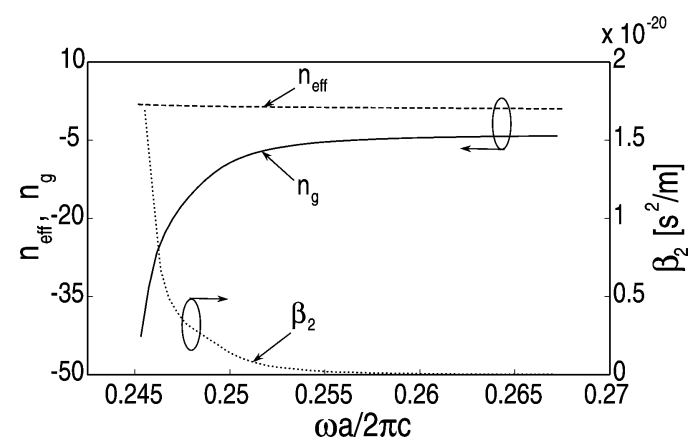

Fig. 3. Dependence of $n_{\mathrm{eff}}, n_{g}$, and $\beta_{2}$ on the normalized frequency determined for a Si-PhCW with $h=0.6 a, r=0.22 a$, and $a=412 \mathrm{~nm}$

the GV of a pulse with frequency $\omega$, whereas $\beta_{2} \equiv \partial^{2} \beta / \partial \omega^{2}$ describes the pulse GVD. The frequency dependence of these dispersion coefficients, calculated for the fundamental mode, are shown in Fig. 3. Among other things, this figure shows that near the Brillouin edge, the group index $n_{g}=c / v_{g}$ has large absolute value ( $v_{g}$ approaches zero asymptotically); an immediate consequence of this dependence being that $\beta_{2}$ becomes very large in this slow-light frequency region. On the other hand, the effective index, defined as $n_{\text {eff }}=\beta c / \omega$, shows small frequency dispersion. Also, note that the GV of this fundamental mode is negative. It is worth mentioning that this same property of propagating modes in PhCs leads to a negative index of refraction and negative refraction in these structures.

\section{DeRIVATION OF COUPLED-Mode EQuATIONS}

In this section, we derive the coupled-mode equations describing the propagation of an optical pulse in a Si-PhCW as well the pulse interaction with the photogenerated FCs.

\section{A. Modes of PhC Waveguides}

The electromagnetic field of guiding modes with frequency $\omega$ is described by the Maxwell equations, which in the frequency domain are written as

$$
\begin{aligned}
& \nabla \times \mathbf{E}(\mathbf{r}, \omega)=i \omega \mu \mathbf{H}(\mathbf{r}, \omega) \\
& \nabla \times \mathbf{H}(\mathbf{r}, \omega)=-i \omega \epsilon_{c}(\mathbf{r}, \omega) \mathbf{E}(\mathbf{r}, \omega)-i \omega \mathbf{P}_{\text {pert }}(\mathbf{r}, \omega)
\end{aligned}
$$

where $\mu$ is the magnetic permeability (here, we set $\mu=\mu_{0}$ ), $\epsilon_{c}(\mathbf{r}, \omega)$ is the dielectric constant of the $\mathrm{PhC}, \mathbf{E}$ and $\mathbf{H}$ are the electric and magnetic fields, respectively, and $\mathbf{P}_{\text {pert }}$ is a perturbation polarization, which, in our case, is generated by the FCs and nonlinear (Kerr) effects.

Let us assume that, at the frequency $\omega$, the unperturbed $\mathrm{PhC}$ waveguide $\left(\mathbf{P}_{\text {pert }}=0\right)$ has $M$ guiding modes. Based on the Bloch theorem, the fields of these modes can be written as

$$
\begin{array}{ll}
\mathbf{E}_{m \sigma}(\mathbf{r}, \omega)=\mathbf{e}_{m \sigma}(\mathbf{r}, \omega) e^{i \sigma \beta_{m} z}, & m=1,2, \ldots, M \\
\mathbf{H}_{m \sigma}(\mathbf{r}, \omega)=\mathbf{h}_{m \sigma}(\mathbf{r}, \omega) e^{i \sigma \beta_{m} z}, & m=1,2, \ldots, M
\end{array}
$$

where $\beta_{m}$ is the mode propagation constant and $\sigma=+(\sigma=-)$ denotes forward (backward) propagating modes; here, we assume that the fields depend on time as $e^{-i \omega t}$. The fields $\mathbf{e}_{m \sigma}$ and $\mathbf{h}_{m \sigma}$ are periodic along the $z$-axis, with period $a$. Moreover, the forward and backward propagating modes obey the following symmetry relations:

$$
\begin{aligned}
\mathbf{e}_{m-}(\mathbf{r}, \omega) & =\mathbf{e}_{m+}^{*}(\mathbf{r}, \omega) \\
\mathbf{h}_{m-}(\mathbf{r}, \omega) & =-\mathbf{h}_{m+}^{*}(\mathbf{r}, \omega)
\end{aligned}
$$

and thus, in practice, one has to determine only the forward propagating modes. In addition, the guiding modes satisfy the following orthogonality relation:

$$
\frac{1}{4} \int_{S}\left(\mathbf{e}_{m \sigma} \times \mathbf{h}_{m^{\prime} \sigma^{\prime}}^{*}+\mathbf{e}_{m^{\prime} \sigma^{\prime}}^{*} \times \mathbf{h}_{m \sigma}\right) \cdot \hat{\mathbf{z}} d S=\sigma P_{m} \delta_{\sigma \sigma^{\prime}} \delta_{m m^{\prime}}
$$

where $P_{m}$ is the power carried by the mode $m$. Note that the waveguide modes (3) are exact solutions of the unperturbed Maxwell equation (2), and thus, they should not be confused with the so-called local modes, which have been used to describe, e.g., wave propagation in tapered waveguides [31] or pulse copropagation in 1-D Bragg gratings [32].

\section{B. Perturbations of the PhC Waveguide}

When optical pulses propagate in a $\mathrm{Si}-\mathrm{PhCW}$, its optical properties are changed via a local variation of the dielectric constant $\delta \epsilon(\mathbf{r})$. This perturbative effect, which is described by the polarization $\mathbf{P}_{\text {pert }}$ in (2b), has two sources: the linear change of the dielectric constant via generation of FCs and a nonlinearly induced variation of the index of refraction through the Kerr effect. The linear contribution to $\mathbf{P}_{\text {pert }}, \delta \mathbf{P}_{\text {lin }}(\mathbf{r})$, can be written as

$$
\delta \mathbf{P}_{\text {lin }}(\mathbf{r}, t)=\delta \epsilon_{\mathrm{FC}}(\mathbf{r}) \mathbf{E}(\mathbf{r}, t)
$$

where [3]

$$
\delta \epsilon_{\mathrm{FC}}(\mathbf{r})=\left(2 \epsilon_{0} n \delta n_{\mathrm{FC}}+i \frac{\epsilon_{0} c n}{\omega} \alpha_{\mathrm{FC}}\right) \Sigma(\mathbf{r}) .
$$

Here, $\Sigma(\mathbf{r})$ is the characteristic function of the domain where FCs can be generated ( $\Sigma=1$ in the domain occupied by Si and $\Sigma=0$, otherwise). Based on the Drude model, the FC-induced change of the index of refraction $\delta n_{\mathrm{FC}}$ and FC losses $\alpha_{\mathrm{FC}}$ are given by [33]

$$
\begin{aligned}
\delta n_{\mathrm{FC}} & =-\frac{e^{2}}{2 \epsilon_{0} n \omega^{2}}\left(\frac{N_{e}}{m_{\mathrm{ce}}^{*}}+\frac{N_{h}}{m_{\mathrm{ch}}^{*}}\right) \\
\alpha_{\mathrm{FC}} & =\frac{e^{3}}{\epsilon_{0} c n \omega^{2}}\left(\frac{N_{e}}{\mu_{e} m_{\mathrm{ce}}^{*}}+\frac{N_{h}}{\mu_{h} m_{\mathrm{ch}}^{*}}\right) .
\end{aligned}
$$

Here, $e$ is the charge of the electron, $\mu_{e}\left(\mu_{h}\right)$ is the electron (hole) mobility, $m_{\mathrm{ce}}^{*}=0.26 m_{0}\left(m_{\mathrm{ch}}^{*}=0.39 m_{0}\right)$ is the conductivity effective mass of the electrons (holes), with $m_{0}$ the mass of the electron, and $N_{e}\left(N_{h}\right)$ is the induced variation of the electrons (holes) density (in what follows, we consider $N_{e}=N_{h} \equiv N$ ).

The nonlinear contribution to $\mathbf{P}_{\text {pert }}, \delta \mathbf{P}_{\text {Kerr }}(\mathbf{r})$, is described by a third-order nonlinear susceptibility, $\hat{\chi}^{(3)}(\mathbf{r})$, which can be written as

$$
\delta \mathbf{P}_{\text {Kerr }}(\mathbf{r}, t)=\epsilon_{0} \hat{\chi}^{(3)}(\mathbf{r}) \vdots \mathbf{E}(\mathbf{r}, t) \mathbf{E}(\mathbf{r}, t) \mathbf{E}(\mathbf{r}, t) .
$$

The real part of the susceptibility $\hat{\chi}^{(3)}$ describes parametric processes of photon scattering, e.g., SPM and XPM, which lead 
to a change in the index of refraction, while the imaginary part of $\hat{\chi}^{(3)}$ corresponds to two-photon absorption (TPA). Moreover, since Si belongs to the crystallographic point group $m 3 m$, the susceptibility tensor $\hat{\chi}^{(3)}$ has 21 nonzero elements, of which only four are independent, namely, $\chi_{1111}, \chi_{1122}, \chi_{1212}$, and $\chi_{1221}$ [34]. In addition, for picoseconds pulses considered here, the frequency dispersion of the nonlinear susceptibility can be neglected, so that the Kleinman symmetry relations imply that the last three elements are equal. Also, a recent experiment has shown that $\hat{\chi}_{1111}^{(3)}=2.36 \hat{\chi}_{1122}^{(3)}$ [35] within a broad frequency range, and thus, in the end, the Kerr nonlinearity is characterized by only one element of the tensor $\hat{\chi}^{(3)}$.

\section{Coupled-Mode Equation for the Optical Field}

In order to derive the coupled-mode equation describing pulse propagation in $\mathrm{Si}-\mathrm{PhCW}$, we employ an approach based on the conjugated form of the Lorentz reciprocity theorem [3], [36]-[38]. To this end, let us consider two sets of solutions of the Maxwell equation (2), $\left[\mathbf{E}_{1}\left(\mathbf{r}, \omega_{1}\right), \mathbf{H}_{1}\left(\mathbf{r}, \omega_{1}\right)\right]$ and $\left[\mathbf{E}_{2}\left(\mathbf{r}, \omega_{2}\right), \mathbf{H}_{2}\left(\mathbf{r}, \omega_{2}\right)\right]$, which correspond to the dielectric constant $\epsilon_{1}\left(\mathbf{r}, \omega_{1}\right)$ and $\epsilon_{2}\left(\mathbf{r}, \omega_{2}\right)$, respectively. If we insert the vector $\mathbf{F}=\mathbf{E}_{2} \times \mathbf{H}_{1}^{*}+\mathbf{E}_{1}^{*} \times \mathbf{H}_{2}$ in the integral identity

$$
\int_{S} \nabla \cdot \mathbf{F} d S=\frac{\partial}{\partial z} \int_{S} \mathbf{F} \cdot \hat{\mathbf{z}} d S+\oint_{\partial S} \mathbf{F} \cdot \mathbf{n} d l
$$

where $S$ is the transverse section at position $z$, and use the Maxwell equation, we arrive at the following relation:

$$
\begin{aligned}
\frac{\partial}{\partial z} \int_{S} \mathbf{F} \cdot \hat{\mathbf{z}} d S= & -\oint_{\partial S} \mathbf{F} \cdot \mathbf{n} d l+i \mu_{0}\left(\omega_{2}-\omega_{1}\right) \int_{S} \mathbf{H}_{1}^{*} \cdot \mathbf{H}_{2} d S \\
& +i \int_{S}\left(\omega_{2} \epsilon_{2}-\omega_{1} \epsilon_{1}\right) \mathbf{E}_{1}^{*} \cdot \mathbf{E}_{2} d S
\end{aligned}
$$

Now, as the first set of fields, let us consider a mode of the unperturbed waveguide $\left(\mathbf{P}_{\text {pert }}=0\right)$, which corresponds to the frequency $\omega_{1}=\bar{\omega}(\bar{\omega}$ is a reference frequency, which can be chosen to be the central frequency of the pulse):

$$
\begin{aligned}
& \mathbf{E}_{1}(\mathbf{r}, \bar{\omega})=\frac{\mathbf{e}_{n \rho}(\mathbf{r}, \bar{\omega})}{\sqrt{P_{n}}} e^{i \rho \bar{\beta}_{n} z} \\
& \mathbf{H}_{1}(\mathbf{r}, \bar{\omega})=\frac{\mathbf{h}_{n \rho}(\mathbf{r}, \bar{\omega})}{\sqrt{P_{n}}} e^{i \rho \bar{\beta}_{n} z} .
\end{aligned}
$$

Here, and in what follows, a bar over a symbol means that the corresponding quantity is evaluated at $\bar{\omega}$. Furthermore, as the second set of fields, we take the actual fields that propagate in the perturbed waveguide at the frequency $\omega_{2}=\omega$. These fields are written as a series expansion of the guiding modes at frequency $\bar{\omega}$, thus neglecting the frequency dispersion of the modes and the radiative modes. This represents a valid approximation as long as the optical pulse has a narrow spectrum, i.e., $\omega$ is close to $\bar{\omega}$. Thus, the second set of fields are expanded as

$$
\begin{aligned}
& \mathbf{E}_{2}(\mathbf{r}, \omega)=\sum_{m \sigma} a_{m \sigma}(z, \omega) \frac{\mathbf{e}_{m \sigma}(\mathbf{r}, \bar{\omega})}{\sqrt{P_{m}}} e^{i \sigma \bar{\beta}_{m} z} \\
& \mathbf{H}_{2}(\mathbf{r}, \omega)=\sum_{m \sigma} a_{m \sigma}(z, \omega) \frac{\mathbf{h}_{m \sigma}(\mathbf{r}, \bar{\omega})}{\sqrt{P_{m}}} e^{i \sigma \bar{\beta}_{m} z} .
\end{aligned}
$$

With the normalization used in (13), the mode amplitudes $a_{m \sigma}(z, \omega)$ are measured in units of $\sqrt{\mathrm{W}}$. Moreover, the dielectric constants in the two cases are $\epsilon_{1}=\bar{\epsilon}_{c}(\mathbf{r})$ and $\epsilon_{2}=$ $\epsilon_{c}(\mathbf{r}, \omega)+\delta \epsilon(\mathbf{r}, \omega)$, where $\epsilon_{c}(\mathbf{r}, \omega)$ is the dielectric constant of the unperturbed $\mathrm{PhC}$. If the material dispersion is neglected, $\epsilon_{c}(\mathbf{r}, \omega)=\bar{\epsilon}_{c}(\mathbf{r})$. Inserting the fields (12) and (13) in (11), and neglecting the line integral in (11), which cancels for guiding modes, one obtains the following set of coupled equation:

$$
\begin{aligned}
\rho \frac{\partial a_{n \rho}}{\partial z}= & B_{n \rho} a_{n \rho}+\sum_{\substack{m \neq n \\
\sigma \neq \rho}} D_{n \rho, m \sigma} a_{m \sigma} \\
& +\frac{i \omega e^{-i \rho \bar{\beta}_{n} z}}{4 \sqrt{P_{n}}} \int_{S} \overline{\mathbf{e}}_{n \rho}^{*} \cdot \mathbf{P}_{\text {pert }}(\mathbf{r}, \omega) d S
\end{aligned}
$$

where

$$
\begin{aligned}
B_{n \rho}= & \frac{i}{4 P_{n}} \int_{S}\left[\mu_{0}(\omega-\bar{\omega})\left|\mathbf{h}_{n \rho}\right|^{2}+\left(\omega \epsilon_{c}-\bar{\epsilon}_{c} \bar{\omega}\right)\left|\mathbf{e}_{n \rho}\right|^{2}\right] d S \\
D_{n \rho, m \sigma}= & \frac{i e^{i\left(\sigma \bar{\beta}_{m}-\rho \bar{\beta}_{n}\right) z}}{4 \sqrt{P_{n} P_{m}}} \int_{S}\left[\mu_{0}(\omega-\bar{\omega}) \mathbf{h}_{m \sigma} \cdot \mathbf{h}_{n \rho}^{*}\right. \\
& \left.+\left(\omega \epsilon_{c}-\bar{\epsilon}_{c} \bar{\omega}\right) \mathbf{e}_{m \sigma} \cdot \mathbf{e}_{n \rho}^{*}\right] d S .
\end{aligned}
$$

Note that in deriving the LHS of (14), we have used the orthogonality relation (5).

The time-dependent fields are obtained by summing over all frequency components contained in the pulse spectrum, i.e.,

$$
\begin{aligned}
\mathbf{E}(\mathbf{r}, t) & =\frac{1}{2} \int_{0}^{\infty} \sum_{m \sigma} a_{m \sigma}(z, \omega) \frac{\mathbf{e}_{m \sigma}(\mathbf{r}, \bar{\omega})}{\sqrt{P_{m}}} e^{i\left(\sigma \bar{\beta}_{m} z-\omega t\right)} d \omega+c . c . \\
& \equiv \frac{1}{2}\left[\mathbf{E}^{(+)}(\mathbf{r}, t)+\mathbf{E}^{(-)}(\mathbf{r}, t)\right] \\
\mathbf{H}(\mathbf{r}, t) & =\frac{1}{2} \int_{0}^{\infty} \sum_{m \sigma} a_{m \sigma}(z, \omega) \frac{\mathbf{h}_{m \sigma}(\mathbf{r}, \bar{\omega})}{\sqrt{P_{m}}} e^{i\left(\sigma \bar{\beta}_{m} z-\omega t\right)} d \omega+c . c . \\
& \equiv \frac{1}{2}\left[\mathbf{H}^{(+)}(\mathbf{r}, t)+\mathbf{H}^{(-)}(\mathbf{r}, t)\right]
\end{aligned}
$$

where $\mathbf{E}^{(+)}(\mathbf{r}, t), \mathbf{H}^{(+)}(\mathbf{r}, t)$ and $\mathbf{E}^{(-)}(\mathbf{r}, t), \mathbf{H}^{(-)}(\mathbf{r}, t)$ are the positive and negative frequency parts of the spectrum, respectively. If we introduce now the pulse envelope in the time domain $A_{n \rho}(z, t)$ as

$$
A_{n \rho}(z, t)=\int_{0}^{\infty} a_{n \rho}(z, \omega) e^{-i(\omega-\bar{\omega}) t} d \omega
$$

then the time-dependent fields (16) become

$$
\begin{aligned}
& \mathbf{E}(\mathbf{r}, t)=\frac{1}{2} \sum_{m \sigma} A_{m \sigma}(z, t) \frac{\mathbf{e}_{m \sigma}(\mathbf{r}, \bar{\omega})}{\sqrt{P_{m}}} e^{i\left(\sigma \bar{\beta}_{m} z-\bar{\omega} t\right)}+c . c . \\
& \mathbf{H}(\mathbf{r}, t)=\frac{1}{2} \sum_{m \sigma} A_{m \sigma}(z, t) \frac{\mathbf{h}_{m \sigma}(\mathbf{r}, \bar{\omega})}{\sqrt{P_{m}}} e^{i\left(\sigma \bar{\beta}_{m} z-\bar{\omega} t\right)}+c . c .
\end{aligned}
$$


Similarly to the fields (16), the time-dependent nonlinear polarization can be written as

$$
\begin{aligned}
\delta \mathbf{P}_{\text {Kerr }}(\mathbf{r}, t) & =\frac{1}{2} \int_{0}^{\infty} \delta \mathbf{P}_{\text {Kerr }}(\mathbf{r}, \omega) e^{-i \omega t} d \omega+c . c . \\
& =\frac{1}{2}\left[\delta \mathbf{P}_{\text {Kerr }}^{(+)}(\mathbf{r}, t)+\delta \mathbf{P}_{\text {Kerr }}^{(-)}(\mathbf{r}, t)\right] .
\end{aligned}
$$

Using this equation and (16), one can cast (9) in the following form:

$$
\delta \mathbf{P}_{\text {Kerr }}^{(+)}(\mathbf{r}, t)=\frac{3}{4} \epsilon_{0} \hat{\chi}^{(3)}(\mathbf{r}) \vdots \mathbf{E}^{(+)}(\mathbf{r}, t) \mathbf{E}^{(-)}(\mathbf{r}, t) \mathbf{E}^{(+)}(\mathbf{r}, t) .
$$

Now we transform (14) in the time domain. To begin with, we expand the coefficients $B_{n \rho}$ and $D_{n \rho, m \sigma}$ in Taylor series, around the frequency $\bar{\omega}$, as

$$
\begin{aligned}
& B_{n \rho}=\left.\sum_{q \geq 1} \frac{\Delta \omega^{q}}{q !} \frac{\partial^{q} B_{n \rho}}{\partial \omega^{q}}\right|_{\omega=\bar{\omega}}=i \sum_{q \geq 1} \frac{\beta_{n \rho}^{(q)}}{q !} \Delta \omega^{q} \\
& D_{n \rho, m \sigma}=\left.\sum_{q \geq 1} \frac{\Delta \omega^{q}}{q !} \frac{\partial^{q} D_{n \rho, m \sigma}}{\partial \omega^{q}}\right|_{\omega=\bar{\omega}}=i \sum_{q \geq 1} \frac{\beta_{n \rho, m \sigma}^{(q)}}{q !} \Delta \omega^{q}
\end{aligned}
$$

where $\Delta \omega=\omega-\bar{\omega}$. Then, we multiply (14) by $e^{-i(\omega-\bar{\omega}) t}$ and integrate over all positive frequencies; the result represents the time-domain coupled-mode equation describing the field envelopes $A_{n \rho}(z, t)$

$$
\begin{aligned}
\rho \frac{\partial A_{n \rho}}{\partial z}= & i \sum_{q \geq 1} \frac{\beta_{n \rho}^{(q)}}{q !}\left(i \frac{\partial}{\partial t}\right)^{q} A_{n \rho} \\
& +i \sum_{\substack { q \geq 1 \\
\begin{subarray}{c}{m \neq n \\
\sigma \neq \rho{ q \geq 1 \\
\begin{subarray} { c } { m \neq n \\
\sigma \neq \rho } }\end{subarray}} \frac{\beta_{n \rho, m \sigma}^{(q)}}{q !}\left(i \frac{\partial}{\partial t}\right)^{q} A_{m \sigma} \\
& +i \omega\left[\vartheta_{n}(z) A_{n \rho}+\sum_{\substack{m \neq n \\
\sigma \neq \rho}} \vartheta_{n \rho, m \sigma}(z) A_{m \sigma}\right] \\
& +\frac{3 i \omega \epsilon_{0}}{16} \sum_{\vec{m} \vec{\sigma}} \Upsilon_{n \rho, \vec{m} \vec{\sigma}}(z) A_{m_{1} \sigma_{1}} A_{m_{2} \sigma_{2}} A_{m_{3} \sigma_{3}}^{*} .
\end{aligned}
$$

Here, $\vec{m}=\left(m_{1}, m_{2}, m_{3}\right), \vec{\sigma}=\left(\sigma_{1}, \sigma_{2}, \sigma_{3}\right)$, and the linear and nonlinear coupling coefficients are given by

$$
\begin{aligned}
\vartheta_{n}(z)= & \frac{1}{4 P_{n}} \int_{S} \delta \epsilon_{F C}(\mathbf{r})\left|\mathbf{e}_{n \rho}\right|^{2} d S \\
\vartheta_{n \rho, m \sigma}(z)= & \frac{e^{i\left(\sigma \beta_{m}-\rho \beta_{n}\right) z}}{4 \sqrt{P_{n} P_{m}}} \int_{S} \delta \epsilon_{F C}(\mathbf{r}) \mathbf{e}_{n \rho}^{*} \cdot \mathbf{e}_{m \sigma} d S \\
\Upsilon_{n \rho, \vec{m} \vec{\sigma}}(z)= & \frac{e^{i\left(\sigma_{1} \beta_{m_{1}}+\sigma_{2} \beta_{m_{2}}-\sigma_{3} \beta_{m_{3}}-\rho \beta_{n}\right) z}}{\sqrt{P_{n} P_{m_{1}} P_{m_{2}} P_{m_{3}}}} \\
& \times \int_{S} \mathbf{e}_{n \rho}^{*} \cdot \hat{\chi}^{(3)}(\mathbf{r}): \mathbf{e}_{m_{1} \sigma_{1}} \mathbf{e}_{m_{2} \sigma_{2}} \mathbf{e}_{m_{3} \sigma_{3}}^{*} d S .
\end{aligned}
$$

Note that in deriving (22), we have neglected the frequency dispersion of the FC-induced change in the dielectric constant $\delta \epsilon_{\mathrm{FC}}$ and the frequency dependence of the coefficient in the nonlinear term in (14); also, for convenience, in (22), we replaced $\bar{\omega}$ with $\omega$. Moreover, since $\delta \epsilon_{\mathrm{FC}}$ and $\hat{\chi}^{(3)}(\mathbf{r})$ vanish outside the domain occupied by the Si slab, the integrals in (23) are, in fact, performed only over the Si transverse area $S_{n l}$.

The first two terms on the RHS of (22) describe the mode dispersion and the frequency dispersion of the mode coupling constant, respectively. The next two terms correspond to the FCinduced mode dispersion and the FC-mediated mode coupling, respectively. Generally, the latter effect is small due to the mode mismatch; however, this effect can become significant in the case of coupling between forward and backward propagating modes, where wave vectors are close to the edge of the Brillouin zone, i.e., for slow-light modes.

\section{Carrier Dynamics}

In order to determine the carrier dynamics, we first find the rate at which $e-h$ pairs are generated optically, via the TPA. Thus, we multiply (22) by $A_{n \rho}^{*}$, multiply the complex conjugate of (22) by $A_{n \rho}$, and sum them; the result can be cast as

$$
\begin{aligned}
& \frac{\partial}{\partial z} \sum_{n \rho}\left|A_{n \rho}\right|^{2} \\
& =-\frac{3 \omega \epsilon_{0}}{8} \mathfrak{I m}\left[\sum_{n \rho, \vec{m} \vec{\sigma}} \Upsilon_{n \rho, \vec{m} \vec{\sigma}}(z) \times A_{m_{1} \sigma_{1}} A_{m_{2} \sigma_{2}} A_{m_{3} \sigma_{3}}^{*} A_{n \rho}^{*}\right]
\end{aligned}
$$

The sum on the LHS of (24) represents the total optical power transferred to the FCs, within the distance $d z$. To calculate the corresponding infinitesimal volume $d V$ in which the carriers are generated as a result of this energy transfer, we use the time average of the Poynting vector to define the effective area of the field inside the Si slab

$$
A_{n l}(z)=\frac{\left[\int_{S_{n l}}\left|\langle\mathbf{E}(\mathbf{r}, t) \times \mathbf{H}(\mathbf{r}, t)\rangle_{t}\right| d S\right]^{2}}{\int_{S_{n l}}\left|\langle\mathbf{E}(\mathbf{r}, t) \times \mathbf{H}(\mathbf{r}, t)\rangle_{t}\right|^{2} d S} .
$$

Then, $d V=A_{n l} d z$. In (25), $\langle f\rangle_{t}$ means the time average of $f$. Using (18) and assuming that $A_{n \rho}$ varies much slower than $e^{-i \omega t}$, one can cast (25) in the following form:

$$
A_{n l}(z)=\frac{\left[\sum_{n \rho} \frac{\left|A_{n \rho}\right|^{2}}{P_{n}} \int_{S_{n l}}\left|\mathfrak{R e}\left(\mathbf{e}_{n \rho} \times \mathbf{h}_{n \rho}^{*}\right)\right| d S\right]^{2}}{\sum_{n \rho} \frac{\left|A_{n \rho}\right|^{4}}{P_{n}^{2}} \int_{S_{n l}}\left|\mathfrak{R e}\left(\mathbf{e}_{n \rho} \times \mathbf{h}_{n \rho}^{*}\right)\right|^{2} d S} .
$$

In practice, it is rather cumbersome to work with the expression (26), so that, since the waveguide modes are primarily concentrated within the domain of the missing row of holes, we can simply approximate $A_{n l} \approx a h$. In addition, in the next section, we will show that in cases of practical interest, (26) simplifies considerably.

Since the electron-hole pairs are generated via TPA, for the creation of each such pair, it is required an amount of energy that is equal to $2 \hbar \omega$. As a result, the carriers dynamics is governed 
by the following rate equation:

$$
\begin{aligned}
\frac{\partial N}{\partial t}= & -\frac{N}{\tau_{c}}+\frac{3 \epsilon_{0}}{16 \hbar A_{n l}} \mathfrak{I m} \\
& \times\left[\sum_{n \rho, \vec{m} \vec{\sigma}} \Upsilon_{n \rho, \vec{m} \vec{\sigma}}(z) \times A_{m_{1} \sigma_{1}} A_{m_{2} \sigma_{2}} A_{m_{3} \sigma_{3}}^{*} A_{n \rho}^{*}\right]
\end{aligned}
$$

where $\tau_{c}$ is the relaxation time. Recent experimental work has shown that $\tau_{c}$ is of the order of $1 \mathrm{~ns}$ [39], although numerical simulations of carrier diffusion suggest that it can be as small as 10 ps [40].

The system of coupled equation (22) and (27) fully describes the dynamics of the optical field and FCs, and represents the main result derived in this paper.

\section{Application to Single-Mode WaVeguides AND COUPLING OF Two MODES}

In this section, we will analyze in more detail two cases of practical interest, namely, the pulse propagation in a singlemode $\mathrm{Si}-\mathrm{PhCW}$ and the interaction between two pulses propagating in different $\mathrm{PhC}$ waveguide modes.

\section{A. Single-Mode Waveguide}

If the waveguide supports a single mode, the optical field is described by a single envelope function $A(z, t)$. Moreover, using (15a) and (21a), we write the dispersion coefficients as

$$
\begin{aligned}
& \beta^{(1)}(z)=\frac{1}{4 P} \int_{S}\left[\mu_{0}|\mathbf{h}(\mathbf{r})|^{2}+\frac{\partial}{\partial \omega}\left(\omega \epsilon_{c}\right)|\mathbf{e}(\mathbf{r})|^{2}\right] d S \\
& \beta^{(n)}(z)=\frac{\partial^{n-1} \beta^{(1)}(z)}{\partial \omega^{n-1}}, \quad n \geq 2 .
\end{aligned}
$$

We can use the relationship between the mode power $P$ and the mode energy $W$ to relate the parameters $\beta^{(1)}(z)$ and $\beta^{(2)}(z)$ to the GV and GVD coefficients, respectively. Thus, for a $\mathrm{PhC}$ mode, the following relations hold:

$$
P=\frac{W_{e}+W_{m}}{a} v_{g}=\frac{2 W_{e}}{a} v_{g}=\frac{2 W_{m}}{a} v_{g}
$$

where

$$
\begin{aligned}
& W_{e}=\frac{1}{4} \int_{V_{\text {cell }}} \frac{\partial}{\partial \omega}\left(\omega \epsilon_{c}\right)|\mathbf{e}(\mathbf{r})|^{2} d V \\
& W_{m}=\frac{1}{4} \int_{V_{\text {cell }}} \mu_{0}|\mathbf{h}(\mathbf{r})|^{2} d V
\end{aligned}
$$

and we used the fact that the mode contains the same amount of electric and magnetic energy. Then, (28) become

$$
\begin{aligned}
& \beta^{(1)}(z)=\frac{\delta(z)}{v_{g}} \\
& \beta^{(n)}(z)=\frac{\partial^{n-1}}{\partial \omega^{n-1}}\left[\frac{\delta(z)}{v_{g}}\right], \quad n \geq 2
\end{aligned}
$$

where

$$
\delta(z)=\frac{a \int_{S}\left[\mu_{0}|\mathbf{h}(\mathbf{r})|^{2}+\frac{\partial}{\partial \omega}\left(\omega \epsilon_{c}\right)|\mathbf{e}(\mathbf{r})|^{2}\right] d S}{\int_{V_{\text {cell }}}\left[\mu_{0}|\mathbf{h}(\mathbf{r})|^{2}+\frac{\partial}{\partial \omega}\left(\omega \epsilon_{c}\right)|\mathbf{e}(\mathbf{r})|^{2}\right] d V} .
$$
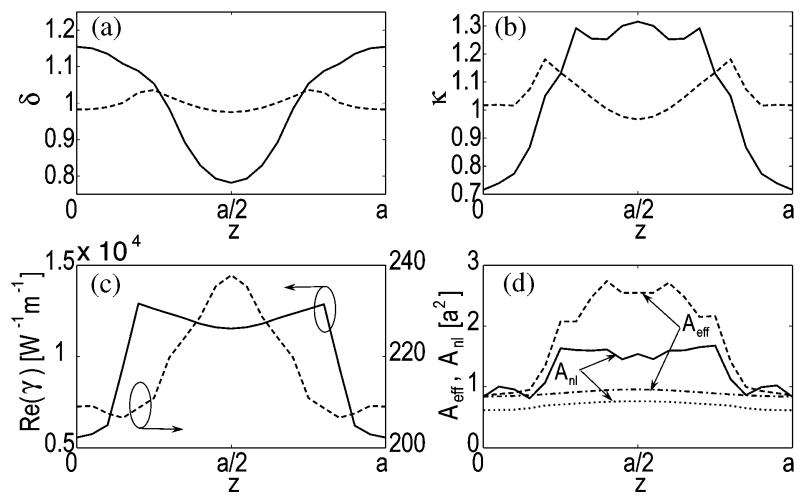

Fig. 4. Dependence of Si-PhCW parameters on diatance $z$ determined for the fast- and slow-light modes in Fig. 2. Dashed and solid curves in (a)-(c) correspond to the fast- and slow-light modes, respectively. (d) Dashed and solid curves correspond to the slow-light mode, and dotted and dashed-dotted curves to the fast-light mode.

Note that (32) shows that the average of $\delta(z)$ over one lattice cell is equal to 1 , i.e.,

$$
\langle\delta\rangle_{z} \equiv \frac{1}{a} \int_{z}^{z+a} \delta\left(z^{\prime}\right) d z^{\prime}=1 .
$$

Moreover, as (28) shows, if we neglect the frequency dispersion of $\delta$, the higher order dispersion coefficients can be written as $\beta^{(n)}(z)=\beta_{n} \delta(z)$. In Fig. 4(a), we plot the function $\delta(z)$, calculated for a slow-light mode, with $v_{g}=c / 35$ and $\tilde{\omega}=0.245$, and a mode with $v_{g}=c / 4.125$ and $\tilde{\omega}=0.267$. Note that the variations of $\delta(z)$ are significantly larger in the case of the slow-light mode, as compared to the regular mode, a result that is chiefly explained by the increased spatial extent of the slow-light mode (see Fig. 2).

Similar to the case of uniform Si waveguides [6], we introduce a parameter $\kappa(z)$ that characterizes the overlap between the mode and the Si domain, where FCs are generated as

$$
\kappa(z)=\frac{a n^{2} \int_{S_{n l}}|\mathbf{e}(\mathbf{r})|^{2} d S}{\int_{V_{\text {cell }}} \frac{\partial}{\partial \omega}\left(\omega n_{c}^{2}\right)|\mathbf{e}(\mathbf{r})|^{2} d V}
$$

where $V_{\text {cell }}$ is the volume of the primitive cell. Using this definition and substituting (7), (29), and (30a) in (23a), we obtain the following relation:

$$
\vartheta(z)=\left(\frac{\delta n_{F C}}{n v_{g}}+i \frac{c \alpha_{F C}}{2 n \omega v_{g}}\right) \kappa(z) .
$$

The $z$-dependence of the parameter $\kappa$ is presented in Fig. 4(b); again, a much stronger variation of $\kappa$ is observed in the case of the slow-light mode.

The nonlinear effects are characterized by the coefficients $\Upsilon_{n \rho, \vec{m} \vec{\sigma}}(z)$ defined by (23c), which, in the case of a single mode, reduce to a single parameter. Thus, substituting (29) in (23c), one obtains

$$
\begin{aligned}
v(z) & =\frac{a^{2}}{4 W_{e}^{2} v_{g}^{2}} \int_{S_{n l}} \mathbf{e}(\mathbf{r})^{*} \cdot \hat{\chi}^{(3)}(\mathbf{r}): \mathbf{e}(\mathbf{r}) \mathbf{e}(\mathbf{r}) \mathbf{e}^{*}(\mathbf{r}) d S \\
& =\frac{4}{\epsilon_{0}^{2} a^{2} v_{g}^{2}} \Gamma(z) .
\end{aligned}
$$


Here, $\Gamma(z)$ plays the role of an effective nonlinear susceptibility and is defined as

$$
\Gamma(z)=\frac{a^{4} \int_{S_{n l}} \mathbf{e}(\mathbf{r})^{*} \cdot \hat{\chi}^{(3)}(\mathbf{r}): \mathbf{e}(\mathbf{r}) \mathbf{e}(\mathbf{r}) \mathbf{e}^{*}(\mathbf{r}) d S}{\int_{V_{\text {cell }}} \frac{\partial}{\partial \omega}\left(\omega n_{c}^{2}\right)|\mathbf{e}(\mathbf{r})|^{2} d V} .
$$

Note that for Si-PhCWs fabricated along the [110] direction, the crystal principal axes are different from the coordinate axes in which the fields are calculated, so that, in order to calculate $\Gamma(z)$ in (37), one has to transform the tensor $\hat{\chi}^{(3)}$ into the coordinate system of the $\mathrm{PhC}[3]$

$$
\hat{\chi}_{c, i j k l}^{(3)}=\hat{R}_{i \alpha} \hat{R}_{j \beta} \hat{R}_{k \gamma} \hat{R}_{l \delta} \hat{\chi}_{\alpha \beta \gamma \delta}^{(3)}
$$

where $\hat{R}$ is the corresponding rotation matrix. This procedure can also be important when the waveguide is formed along directions other than the $K \Gamma$ symmetry axis of the $\mathrm{PhC}$.

Now we can write down the equation that governs the pulse dynamics in the particular case of a single $\mathrm{PhC}$ waveguide. Thus, using (31), (35), and (36), we can cast (22) in the following form:

$$
\begin{aligned}
i\left[\frac{\partial A}{\partial z}+\frac{\delta(z)}{v_{g}} \frac{\partial A}{\partial t}\right] & -\frac{\beta_{2} \delta(z)}{2} \frac{\partial^{2} A}{\partial t^{2}}=-\frac{\omega \delta n_{F C}}{n v_{g}} \kappa(z) A \\
& -i \frac{c \alpha_{F C}}{2 n v_{g}} \kappa(z) A-\gamma(z)|A|^{2} A
\end{aligned}
$$

where

$$
\gamma(z)=\frac{3 \omega \Gamma(z)}{4 \epsilon_{0} a^{2} v_{g}^{2}}
$$

is the effective nonlinear parameter of the waveguide. The real $\left(\gamma^{\prime}\right)$ and imaginary $\left(\gamma^{\prime \prime}\right)$ parts of $\gamma(z)$ characterize the SPM and TPA, respectively. Note that in the limit case of waveguides whose transverse section does not depend on $z$, (40), in conjunction with (36), reduces to a previously derived formula that characterizes the effective optical nonlinearity of uniform $\mathrm{Si}$ wire waveguides [6]. In Fig. 4(c), we plot the $z$-dependence of $\gamma^{\prime}$, calculated for a slow-light mode and a mode for which $v_{g} \lesssim c$. Importantly, this figure shows that the effective nonlinearity probed by the slow-light mode is almost two orders of magnitude larger than that of the fast-light mode.

Equation (27) that governs the FCs dynamics has a simple form in the case of single-mode waveguides. Thus, combining (27) and (36), we obtain the following equation:

$$
\frac{\partial N(z, t)}{\partial t}=-\frac{N(z, t)}{\tau_{c}}+\frac{3 \Gamma^{\prime \prime}(z)}{4 \epsilon_{0} \hbar a^{2} v_{g}^{2} A_{n l}(z)}|A(z, t)|^{4}
$$

where

$$
A_{n l}(z)=\frac{\left\{\int_{S_{n l}}\left|\mathfrak{R e}\left[\mathbf{e}(\mathbf{r}) \times \mathbf{h}(\mathbf{r})^{*}\right]\right| d S\right\}^{2}}{\int_{S_{n l}}\left|\mathfrak{R e}\left[\mathbf{e}(\mathbf{r}) \times \mathbf{h}(\mathbf{r})^{*}\right]\right|^{2} d S} .
$$

The $z$-dependence of the area $A_{n l}$, expressed in units of $a^{2}$, is shown in Fig. 4(d). In agreement with the mode profiles shown in Fig. 2, the effective transverse area in which FCs are generated is larger in the case of the slow-light mode. For comparison, we also show in Fig. 4(d) the effective area of the mode $A_{\text {eff }}$ defined by (42), but with the integration area $S_{n l}$ replaced by the entire area $S$. As expected, $A_{\text {eff }}>A_{n l}$.
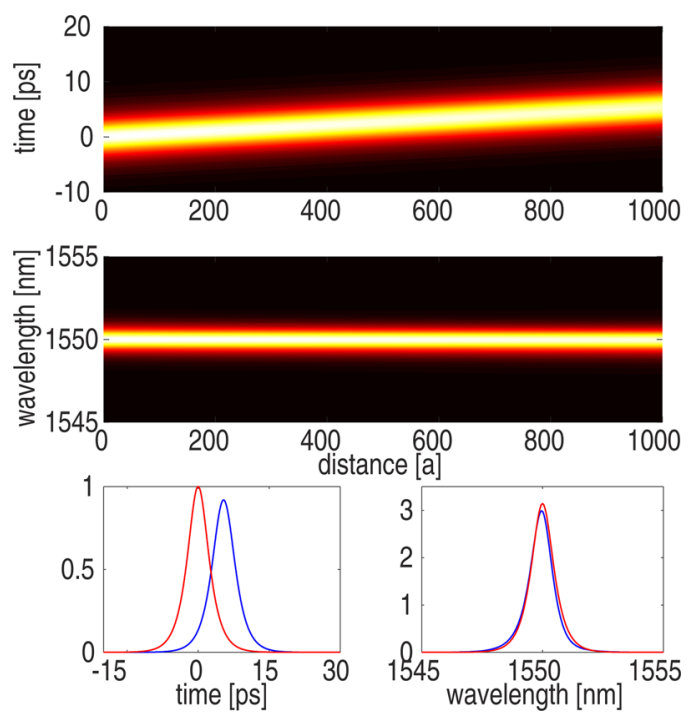

Fig. 5. Top two panels: the temporal and spectral pulse profile versus distance $z$. Bottom panels show the input (red) and output (blue) pulses, both in the time and wavelength domains. The pulse GV $v_{g}=c / 4.125$, which corresponds to $\tilde{\omega}=0.267$. The Si-PhCW has $h=0.6 a$ and $r=0.22 a$, with $a=421 \mathrm{~nm}$. The pulsewidth $T_{0}=2 \mathrm{ps}$, the pulse peak power $P_{0}=3 \mathrm{~W}$, and $\beta_{2}=10 \mathrm{ps}^{2} / \mathrm{m}$.
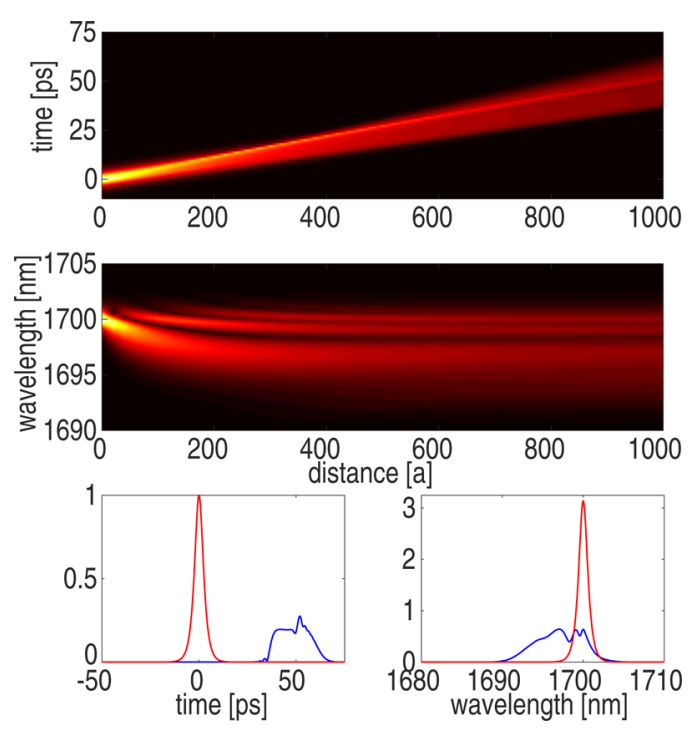

Fig. 6. Same as in Fig. 5, but for a slow-light mode with $v_{g}=c / 35$ and $\tilde{\omega}=0.245$. The pulsewidth $T_{0}=2 \mathrm{ps}$, the pulse peak power $P_{0}=3 \mathrm{~W}$, and $\beta_{2}=10^{4} \mathrm{ps}^{2} / \mathrm{m}$.

Among other things, (39) and (41) suggest that the GV of the pulse would have a strong influence on the pulse dynamics, especially at large peak power. Thus, the linear terms in (39) are proportional to $v_{g}^{-1}$, which implies that the FC-induced losses are proportional to $v_{g}^{-1}$, whereas the nonlinear term depends on the $\mathrm{GV}$ as $v_{g}^{-2}$. However, as the peak power increases, this picture becomes more complicated. As (41) shows, the amount of FCs generated via TPA is proportional to $v_{g}^{-2}$, and thus, the FC-induced losses become proportional to $v_{g}^{-3}$ [see (8b)]. To illustrate this strong dependence of the pulse dynamics on $\mathrm{GV}$, we show in Figs. 5 and 6, the evolution of a pulse that propagates in a Si-PhCW in the fast-light and slow-light regimes, 
respectively. In these numerical simulations, the spatial integration step was $a / 20$ and the relaxation time $\tau_{c}=0.5 \mathrm{~ns}$. In both cases, the pulse parameters, i.e., the pulsewidth $T_{0}$ and pulse peak power $P_{0}$, are the same. These figures show that, in the fast-light regime, the pulse remains almost unchanged during the propagation, except for a small decrease in amplitude due to the FC losses and TPA. However, in the slow-light regime, the pulse shape changes dramatically both in the time as well as the frequency domain. Thus, since in this case, $\beta_{2}$ is three orders of magnitude larger than in the previous case, the temporal width of the pulse increases significantly. In addition, the pulse decay is much stronger in this case, which means that the optical losses due to the generation of FCs and TPA are larger. Importantly, in the slow-light regime, the spectrum of the pulse shows a series of oscillations, a signature of the phase modulation induced by the FC dispersion and the Kerr effect. Indeed, the nonlinear coefficient $\gamma$ of the slow-light mode is two orders of magnitude larger than in the case of the fast mode, so that in this case, we expect that the pulse is much strongly influenced by the nonlinear effects and the increased amount of generated FCs.

It is worth mentioning that (39) is similar to the equation describing pulse propagation in optical fiber links whose dispersion and nonlinearity varies periodically with distance. Drawing on this similarity, it is expected that one can use $\mathrm{Si}-\mathrm{PhCW}$ to observe experimentally optical solitons. Specifically, since the characteristic nonlinear and dispersion lengths are much larger than $a$, the corresponding solitons will be similar to the so-called guiding-center solitons [41]-[44].

\section{B. Two-mode Coupling}

Two cases are of interest when considering the coupling of two waveguide modes, namely, coupling of two modes that copropagate in the same direction and the coupling of two counterpropagating modes. Here, we restrict our analysis to the first case; the latter one can be treated in a similar way.

To this end, we note that the formulas for the mode parameters introduced in the previous section, which describe the mode selfinteraction, remain unchanged. Moreover, since the two modes propagate in the same direction, we can choose $\rho_{1}=\rho_{2}=1$. Then, the coefficients that describe the frequency dispersion of the mode coupling and the FC-induced mode coupling can be, respectively, writtenas

$$
\begin{aligned}
& \beta_{12}^{(1)}(z)=\frac{e^{i\left(\beta_{1}-\beta_{2}\right) z}}{4 \sqrt{P_{1} P_{2}}} \int_{S}\left[\mu_{0} \mathbf{h}_{1} \cdot \mathbf{h}_{2}^{*}+\frac{\partial}{\partial \omega}\left(\omega \epsilon_{c}\right) \mathbf{e}_{1} \cdot \mathbf{e}_{2}^{*}\right] d S \\
& \vartheta_{12}(z)=\frac{e^{i\left(\beta_{1}-\beta_{2}\right) z}}{4 \sqrt{P_{1} P_{2}}} \int_{S} \delta \epsilon_{F C}(\mathbf{r}) \mathbf{e}_{1}^{*} \cdot \mathbf{e}_{2} d S
\end{aligned}
$$

From symmetry considerations, it follows that $\beta_{12}^{(1)}=\beta_{21}^{(1) *}$; also, $\vartheta_{21}$ is obtained by swapping in (43b) the indices 1 and 2 . Moreover, the nonlinear effects are determined by the coefficients $v$, which in the particular case of two coupled modes, for the mode 1 , are given by the following relations:

$$
\begin{aligned}
& \Upsilon_{1111}(z)=\frac{1}{P_{1}^{2}} \int_{S_{n l}} \mathbf{e}_{1}^{*} \cdot \hat{\chi}^{(3)}: \mathbf{e}_{1} \mathbf{e}_{1} \mathbf{e}_{1}^{*} d S \\
& \Upsilon_{1112}(z)=\frac{e^{i\left(\beta_{1}-\beta_{2}\right) z}}{P_{1} \sqrt{P_{1} P_{2}}} \int_{S_{n l}} \mathbf{e}_{1}^{*} \cdot \hat{\chi}^{(3)}: \mathbf{e}_{1} \mathbf{e}_{1} \mathbf{e}_{2}^{*} d S \\
& \Upsilon_{1121}(z)=\frac{e^{-i\left(\beta_{1}-\beta_{2}\right) z}}{P_{1} \sqrt{P_{1} P_{2}}} \int_{S_{n l}} \mathbf{e}_{1}^{*} \cdot \hat{\chi}^{(3)}: \mathbf{e}_{1} \mathbf{e}_{2} \mathbf{e}_{1}^{*} d S \\
& \Upsilon_{1122}(z)=\frac{1}{P_{1} P_{2}} \int_{S_{n l}} \mathbf{e}_{1}^{*} \cdot \hat{\chi}^{(3)}: \mathbf{e}_{1} \mathbf{e}_{2} \mathbf{e}_{2}^{*} d S \\
& \Upsilon_{1221}(z)=\frac{e^{-2 i\left(\beta_{1}-\beta_{2}\right) z}}{P_{1} P_{2}} \int_{S_{n l}} \mathbf{e}_{1}^{*} \cdot \hat{\chi}^{(3)}: \mathbf{e}_{2} \mathbf{e}_{2} \mathbf{e}_{1}^{*} d S \\
& \Upsilon_{1222}(z)=\frac{e^{-i\left(\beta_{1}-\beta_{2}\right) z}}{P_{2} \sqrt{P_{1} P_{2}}} \int_{S_{n l}} \mathbf{e}_{1}^{*} \cdot \hat{\chi}^{(3)}: \mathbf{e}_{2} \mathbf{e}_{2} \mathbf{e}_{2}^{*} d S .
\end{aligned}
$$

Because of the symmetry of these equation, $\Upsilon_{1121}=\Upsilon_{1211}$ and $\Upsilon_{1122}=\Upsilon_{1212}$. The coefficients for the second mode are obtained from (44) by swapping the indices 1 and 2.

With these definitions, the coupled-mode equation that describe the dynamics of the optical fields can be written as

$$
\begin{aligned}
& i\left[\frac{\partial A_{1}}{\partial z}+\frac{\delta_{1}(z)}{v_{g, 1}} \frac{\partial A_{1}}{\partial t}\right]-\frac{\beta_{2,1} \delta_{1}(z)}{2} \frac{\partial^{2} A_{1}}{\partial t^{2}}+i \beta_{12}^{(1)}(z) \frac{\partial A_{1}}{\partial t} \\
& =-\omega\left[\vartheta_{1}(z) A_{1}+\vartheta_{12}(z) A_{2}\right]-\frac{3 \omega \epsilon_{0}}{16}\left[\Upsilon_{1111}(z)\left|A_{1}\right|^{2} A_{1}\right. \\
& \quad+2 \Upsilon_{1122}(z)\left|A_{2}\right|^{2} A_{1}+2 \Upsilon_{1121}(z)\left|A_{1}\right|^{2} A_{2}+\Upsilon_{1112}(z) A_{1}^{2} A_{2}^{*} \\
& \left.\quad+\Upsilon_{1221}(z) A_{2}^{2} A_{1}^{*}+\Upsilon_{1222}(z)\left|A_{2}\right|^{2} A_{2}\right] \\
& i\left[\frac{\partial A_{2}}{\partial z}+\frac{\delta_{2}(z)}{v_{g, 2}} \frac{\partial A_{2}}{\partial t}\right]-\frac{\beta_{2,2} \delta_{2}(z)}{2} \frac{\partial^{2} A_{2}}{\partial t^{2}}+i \beta_{21}^{(1)}(z) \frac{\partial A_{2}}{\partial t} \\
& =-\omega\left[\vartheta_{2}(z) A_{2}+\vartheta_{21}(z) A_{1}\right]-\frac{3 \omega \epsilon_{0}}{16}\left[\Upsilon_{2222}(z)\left|A_{2}\right|^{2} A_{2}\right. \\
& \quad+2 \Upsilon_{2211}(z)\left|A_{1}\right|^{2} A_{2}+2 \Upsilon_{2212}(z)\left|A_{2}\right|^{2} A_{1}+\Upsilon_{2221}(z) A_{2}^{2} A_{1}^{*} \\
& \left.\quad+\Upsilon_{2112}(z) A_{1}^{2} A_{2}^{*}+\Upsilon_{2111}(z)\left|A_{1}\right|^{2} A_{1}\right] .
\end{aligned}
$$

A similar system of equation, which describes the pulse propagation in deep nonlinear 1-D gratings, has been derived using the $\mathbf{k} \cdot \mathbf{v}$ expansion [45]. Note that the first two nonlinear terms in (45), which describe the SPM and XPM effects, also appear in the standard coupled-mode equation describing pulse propagation in low-index contrast gratings, whereas the last four terms are specific to deep nonlinear gratings, such as a $\mathrm{Si}-\mathrm{PhCW}$. However, in practical applications, not all nonlinear terms in (45) will affect the pulse propagation, as not all of them are phase-matched. Finally, the carriers dynamics is governed by (27), with the coefficients $\Upsilon$ defined by (44).

In the case of coupling between counterpropagating waves, one has to take into account that $\rho_{1}=-\rho_{2}=1$, which, in turn, implies that in this case, the phase factors in (43) and (44) are obtained by making the transformation $\beta_{2} \rightarrow-\beta_{2}$. Moreover, since the integrals in (43) and (44) are periodic functions of $z$, their Fourier series expansion shows that strong linear and nonlinear mode coupling can be achieved if the mode propagation 
constant is close to the edge of the first Brillouin zone, as in that case, the Bragg condition $\beta=\pi / a$ is readily satisfied.

\section{CONCLUSION}

In conclusion, we have presented a rigorous derivation of the coupled-mode equation that describe the pulse propagation in $\mathrm{Si}-\mathrm{PhCWs}$. We have included in our analysis linear optical effects induced by the waveguide dispersion and mode coupling, and studied their influence on the pulse propagation. In addition, we have incorporated perturbatively in our model linear effects due to the generated FCs, as well as the optical nonlinearity of the $\mathrm{Si}$-PhCW. We have applied the general theoretical model developed here to two particular cases, namely, pulse propagation in a single-mode $\mathrm{Si}-\mathrm{PhCW}$ and the mutual interaction of two pulses propagating in different modes. One important conclusion of this analysis is that the pulse dynamics in the slow-light and fast-light regimes shows remarkably different characteristics. It should also be noted that the theoretical model presented here can be easily extended to pulses that propagate at different frequencies, in which case one can investigate effects such as stimulated Raman scattering, mode switching, or wavelength conversion.

\section{REFERENCES}

[1] K. K. Lee, D. R. Lim, H. C. Luan, A. Agarwal, J. Foresi, and L. C. Kimerling, "Effect of size and roughness on light transmission in a $\mathrm{Si} / \mathrm{SiO}_{2}$ waveguide: Experiments and model," Appl. Phys. Lett., vol. 77, pp. 1617-1619, 2000.

[2] R. U. Ahmad, F. Pizzuto, G. S. Camarda, R. L. Espinola, H. Rao, and R. M. Osgood, "Ultracompact corner-mirrors and $T$-branches in siliconon-insulator," IEEE Photon. Technol. Lett., vol. 14, no. 1, pp. 65-67, Jan. 2002.

[3] X. Chen, N. C. Panoiu, and R. M. Osgood, "Theory of Raman-mediated pulsed amplification in silicon-wire waveguides," IEEE J. Quantum Electron., vol. 42, no. 2, pp. 160-170, Feb. 2006.

[4] E. Dulkeith, F. Xia, L. Schares, W. M. J. Green, and Y. A. Vlasov, "Group index and group velocity dispersion in silicon-on-insulator photonic wires," Opt. Exp., vol. 14, pp. 3853-3863, 2006.

[5] A. C. Turner, C. Manolatou, B. S. Schmidt, M. Lipson, M. A. Foster, J. E. Sharping, and A. L. Gaeta, "Tailored anomalous group-velocity dispersion in silicon channel waveguides," Opt. Exp., vol. 14, pp. 43574362, 2006.

[6] R. M. Osgood, N. C. Panoiu, J. I. Dadap, X. Liu, X. Chen, I.-W. Hsieh, E. Dulkeith, W. M. J. Green, and Y. A. Vlasov, "Engineering nonlinearities in nanoscale optical systems: Physics and applications in dispersionengineered silicon nanophotonic wires," Adv. Opt. Photon., vol. 1, pp. 162-235, 2009.

[7] R. Claps, D. Dimitropoulos, V. Raghunathan, Y. Han, and B. Jalali, "Observation of stimulated Raman amplification in silicon waveguides," Opt. Exp., vol. 11, pp. 1731-1739, 2003.

[8] R. Espinola, J. I. Dadap, R. M. Osgood, S. J. McNab, and Y. A. Vlasov, "Raman amplification in ultrasmall silicon-on-insulator wire waveguides," Opt. Exp., vol. 12, pp. 3713-3718, 2004.

[9] H. Rong, A. Liu, R. Jones, O. Cohen, D. Hak, R. Nicolaescu, A. Fang, and M. Paniccia, "An all-silicon Raman laser," Nature, vol. 433, pp. 292-294, 2005.

[10] M. A. Foster, A. C. Turner, J. E. Sharping, B. S. Schmidt, M. Lipson, and A. L. Gaeta, "Broadband optical parametric gain on a silicon photonic chip," Nature, vol. 441, pp. 960-963, 2006.

[11] A. Liu, R. Jones, L. Liao, D. Samara Rubio, D. Rubin, O. Cohen, R. Nicolaescu, and M. Paniccia, "A high speed silicon optical modulator based on a metal-oxide semiconductor capacitor," Nature, vol. 427, pp. 615-618, 2004.

[12] Q. Xu, B. Shmidt, S. Pradhan, and M. Lipson, "Micrometre-scale silicon electro-optic modulator," Nature, vol. 435, pp. 325-327, 2005.
[13] G. W. Rieger, K. S. Virk, and J. F. Young, "Nonlinear propagation of ultrafast $1.5 \mu$ mpulses in high-index contrast silicon-on-insulator waveguides," Appl. Phys. Lett., vol. 84, pp. 900-902, 2004.

[14] E. Dulkeith, Y. A. Vlasov, X. Chen, N. C. Panoiu, and R. M. Osgood, "Selfphase-modulation in submicron silicon-on-insulator photonic wires," Opt. Exp., vol. 14, pp. 5524-5534, 2006.

[15] R. Dekker, A. Driessen, T. Wahlbrink, C. Moormann, J. Niehusmann, and M. Forst, "Ultrafast Kerr-induced alloptical wavelength conversion in silicon waveguides using $1.5 \mu$ mfemtosecond pulses," Opt. Exp., vol. 14, pp. 8336-8346, 2006.

[16] I.-W. Hsieh, X. Chen, J. I. Dadap, N. C. Panoiu, R. M. Osgood, S. J. McNab, and Y. A. Vlasov, "Crossphase modulation-induced spectral and temporal effects on co-propagating femtosecond pulses in silicon photonic wires," Opt. Exp., vol. 15, pp. 1135-1146, 2007.

[17] R. Espinola, J. I. Dadap, R. M. Osgood, S. McNab, and Y. Vlasov, "C-band wavelength conversion in silicon photonic wire waveguides," Opt. Exp. vol. 13, pp. 4341-4349, 2005.

[18] Q. Lin, J. Zhang, P. M. Fauchet, and G. P. Agrawal, "Ultrabroadband parametric generation and wavelength conversion in silicon waveguides," Opt. Exp., vol. 14, pp. 4786-4799, 2006.

[19] N. C. Panoiu, X. Chen, and R. M. Osgood, "Modulation instability in silicon photonic nanowires," Opt. Lett., vol. 31, pp. 3609-3611, 2006.

[20] J. I. Dadap, N. C. Panoiu, X. Chen, I-W. Hsieh, X. Liu, C. Y. Chou, E. Dulkeith, S. J. McNab, F. Xia, W. M. J. Green, L. Sekaric, Y. A. Vlasov, and R. M. Osgood, "Nonlinear-optical phase modification in dispersionengineered Si photonic wires," Opt. Exp., vol. 16, pp. 1280-1299, 2008.

[21] Q. Lin, O. J. Painter, and G. P. Agrawal, "Nonlinear optical phenomena in silicon waveguides: Modeling and applications," Opt. Exp., vol. 15, pp. 16604-16644, 2007.

[22] M. Soljacic, S. G. Johnson, S. Fan, M. Ibanescu, E. Ippen, and J. D. Joannopoulos, "Photonic-crystal slow-light enhancement of nonlinear phase sensitivity," J. Opt. Soc. Amer. B, Opt. Phys., vol. 19, pp. 2052 2059, 2002.

[23] Y. A. Vlasov, M. O Boyle, H. F. Hamann, and S. J. McNab, "Active control of slow light on a chip with photonic crystal waveguides," Nature, vol. 438, pp. 65-69, 2005.

[24] A. Theocharidis, T. Kamalakis, A. Chipouras, and T. Sphicopoulos, "Linear and nonlinear optical pulse propagation in photonic crystal waveguides near the band edge," IEEE J. Quantum Electron., vol. 44, no. 11, pp. 1020-1027, Nov. 2008.

[25] J. F. McMillan, X. Yang, N. C. Panoiu, R. M. Osgood, and C. W. Wong, "Enhanced stimulated Raman scattering in slow-light photonic crystal waveguides," Opt. Lett., vol. 31, pp. 1235-1237, 2006.

[26] J. F. McMillan, M. Yu, D. L. Kwong, and C. W. Wong, "Observations of spontaneous Raman scattering in silicon slow-light photonic crystal waveguides," Appl. Phys. Lett., vol. 93, pp. 251105-1-251105-3, 2008.

[27] M. Bahl, N. C. Panoiu, and R. M. Osgood, "Nonlinear optical effects in a two-dimensional photonic crystal containing one-dimensional Kerr defects," Phys. Rev. E, Stat. Phys. Plasmas Fluids Relat. Interdiscip. Top., vol. 67, pp. 056604-1-056604-9, 2003.

[28] B. Corcoran, C. Monat, C. Grillet, D. J. Moss, B. J. Eggleton, T. P. White, L. OFaolain, and T. F. Krauss, "Green light emission in silicon through slow-light enhanced third-harmonic generation in photonic crystal waveguides," Nature Photon., vol. 3, pp. 206-210, 2009.

[29] N. C. Panoiu, M. Bahl, and R. M. Osgood, "Optically tunable superprism effect in nonlinear photonic crystals," Opt. Lett., vol. 28, pp. 2503-2505, 2003.

[30] S. G. Johnson and J. D. Joannopoulos, "Block-iterative frequency-domain methods for Maxwell's equation in a plane wave basis," Opt. Exp., vol. 8 , pp. 173-190, 2001.

[31] A. W. Snyder, "Coupling of modes on a tapered dielectric cylinder," IEEE Trans. Microw. Theory Tech., vol. MTT-18, no. 7, pp. 383-392, Jul. 1970.

[32] J. E. Sipe, C. M. de Sterke, and B. J. Eggleton, "Rigorous derivation of coupled mode equation for short, high-intensity grating-coupled, copropagating pulses," J. Mod. Opt., vol. 49, pp. 1437-1452, 2002.

[33] R. A. Soref and B. R. Bennett, "Electrooptical effects in silicon," IEEE J. Quantum Electron., vol. QE-23, no. 1, pp. 123-129, Jan. 1987.

[34] R. W. Boyd, Nonlinear Optics, 3rd ed. San Diego, CA: Academic, 2008

[35] J. Zhang, Q. Lin, G. Piredda, R. W. Boyd, G. P. Agrawal, and P. M. Fauchet, "Anisotropic nonlinear response of silicon in the near-infrared region," Appl. Phys. Lett., vol. 91, pp. 071113-1-071113-3, 2007.

[36] D. Michaelis, U. Peschel, C. Wachter, and A. Brauer, "Reciprocity theorem and perturbation theory for photonic crystal waveguides," Phys. Rev. E Stat. Phys. Plasmas Fluids Relat. Interdiscip. Top., vol. 68, pp. 065601(R)1-065601(R)-4, 2003. 
[37] T. Kamalakis and T. Sphicopoulos, "A new formulation of coupled propagation equation in periodic nanophotonic waveguides for the treatment of Kerr-induced nonlinearities," IEEE J. Quantum Electron., vol. 43, no. 10, pp. 923-933, Oct. 2007.

[38] A. W. Snyder and J. D. Love, Optical Waveguide Theory. London, U.K.: Chapman \& Hall, 1983.

[39] P. E. Barclay, K. Srinivasan, and O. Painter, "Nonlinear response of silicon photonic crystal microresonators excited via an integrated waveguide and fiber taper," Opt. Exp., vol. 13, pp. 801-820, 2005.

[40] T. Tanabe, H. Taniyama, and M. Notomi, "Carrier diffusion and recombination in photonic crystal nanocavity optical switches," J. Lightw. Technol., vol. 26, pp. 1396-1403, 2008.

[41] A. Hasegawa and Y. Kodama, "Guiding-center soliton in optical fibers," Opt. Lett., vol. 15, pp. 1443-1445, 1990.

[42] J. H. B. Nijhof, N. J. Doran, W. Forysiak, and F. M. Knox, "Stable solitonlike propagation in dispersion managed systems with net anomalous, zero and normal dispersion," Electron. Lett., vol. 33, pp. 1726-1727, 1997.

[43] R. Driben and B. A. Malomed, "Split-step solitons in long fiber links," Opt. Commun., vol. 185, pp. 439-456, 2000.

[44] B. A. Malomed, Soliton Management in Periodic Systems. New York: Springer-Verlag, 2006.

[45] C. M. de Sterke, D. G. Salinas, and J. E. Sipe, "Coupled-mode theory for light propagation through deep nonlinear gratings," Phys. Rev. E, Stat. Phys. Plasmas Fluids Relat. Interdiscip. Top., vol. 54, pp. 1969-1989, 1996.

Nicolae C. Panoiu received the B.Sc. and M.S. degrees in physics from the University of Bucharest, Bucharest, Romania, in 1990 and 1992, respectively, and the Ph.D. degree from New York University (NYU), New York, in 2001.

After graduating from NYU, he was a Postdoctoral Fellow in the Department of Applied Physics and Applied Mathematics, Columbia University, New York. He is currently with the Department of Electronic and Electrical Engineering, University College London, London, U.K. His current research interests include silicon photonics, optical properties of photonic nanostructures, physical properties of metamaterials, optical solitons in fibers and quadratic media, and numerical modeling of electromagnetic structures.

Dr. Panoiu is a member of the Optical Society of America (OSA) and the American Physical Society (APS).
James F. McMillan received the B.A.Sc. degree in electrical engineering from the University of Toronto, Toronto, ON, Canada, in 2004, and the M.S. degree in 2006 from Columbia University, New York, where he is currently working toward the Ph.D. degree.

His current research interests include photonic crystal waveguides and slowlight enhancement of light-matter interaction.
Chee Wei Wong (S'03-M'04) received the B.Sc. degree (with highest honors) in mechanical engineering and the B.A. degree (with highest degree) in economics from the University of California at Berkeley, Berkeley, and the M.Sc. degree and the Ph.D. degree in mechanical engineering (optical nanotechnology) from the Massachusetts Institute of Technology (MIT), Cambridge, in 2001 and 2003, respectively.

In 2004, he joined Columbia faculty. From 1996 to 1999, he was at the University of California at Berkeley. During 2003, he was a Postdoctoral Research Associate with the MIT Microphotonics Center. His current research interests include nonlinear and quantum optics in nanophotonics, silicon electronicphotonic circuits and photonic crystals, quantum dot interactions in nanocavities, nanoelectromechanical systems, and nanofabrication. Since 2003, he has been giving more than 30 invited talks. He is the author or coauthor of more than 25 journal articles, 40 conference papers, and two book chapters. He is the holder of six awarded patents and ten pending patents.

Dr. Wong is a member of the American Physical Society (APS), the American Society of Mechanical Engineers (ASME), the Optical Society of America (OSA), and Sigma Xi. He was the recipient of the 2009 3M Faculty Award, the 2008 National Science Foundation (NSF) CAREER Award, and the 2007 Defense Advanced Research Projects Agency (DARPA) Young Faculty Award. 